\title{
Features of manufactured and impact failure of a layered material based on Ti-6Al-4V alloy
}

\author{
A. A. Sarkeeva \\ aigul-05@mail.ru \\ Institute for Metals Superplasticity Problems RAS, 39 S. Khalturin St., Ufa, 450001, Russia
}

\begin{abstract}
Structural methods for controlling the properties of a layered material (LM) based on Ti-6Al-4V alloy are presented in this work. The layered materials were manufactured by diffusion bonding. They consist of seven and thirteen sheets of the thickness about 1.5 and $0.8 \mathrm{~mm}$, respectively. Two methods of assembling the sheets were used: in one case, they were stacked relative to each other so that the rolling direction (RD) in them coincided (LM of type 1), and in the other, they did not match (LM of type 2), the angle between the RD in the neighboring sheets was 0 and 90 degrees, respectively. It was found that the prismatic texture (texture with datum planes oriented perpendicular to the rolling plane) present in the as-received sheets is inherited by the layered material of type 1 that it causes the anisotropy of impact strength. Almost the same distribution of the pole density of basis in the rolling direction and in the transverse direction determines the isotropy of impact strength in the layered material of type 2. A comparative evaluation of the test results has shown that when the crack propagates simultaneously through all layers (the crack divider orientation) the impact strength value is higher for the 7-layer material when the crack propagates sequentially from one layer to another (the crack arrester orientation) it is higher for the 13-layer material. The quantitative assessment of the fracture characteristics made it possible to establish that the decrease in the impact strength of the samples tested in the crack divider orientation with the number of layers increasing is conditioned by decreasing both the crack initiation energy and the crack propagation energy. In contrast, the improvement of impact strength of the samples tested in the crack arrester orientation occurs due to the significant increasing the crack propagation energy at an insignificant decreasing the crack initiation energy.
\end{abstract}

Keywords: layered material, titanium alloy, diffusion bonding, texture, impact strength.

\section{Особенности получения и ударного разрушения слоистого материала на основе титанового сплава ВТ6}

\author{
Саркеева А. А. \\ Институт проблем сверхпластичности металлов РАН, ул. С. Халтурина, 39, Уфа, 450001, Россия
}

В настоящей работе показаны технологические методы управления свойствами слоистого материала (CM) на основе титанового сплава ВТ6. Для исследований методом диффузионной сварки были изготовлены СМ, состоящие из семи и тринадцати листовых заготовок толщиной $\sim 1.5$ и $\sim 0.8$ мм, соответственно. При этом использовали два метода сборки листовых заготовок: в одном случае их укладывали друг относительно друга так, что направление прокатки (НП) в них совпадало (СМ тип 1), а в другом - не совпадало (СМ тип 2), соответственно угол между НП в соседних заготовках составлял $0^{\circ}$ и $90^{\circ}$. Установлено, что призматическая текстура (текстура с плоскостями базиса, ориентированными перпендикулярно плоскости прокатки), присутствующая в листах в состоянии поставки наследуется слоистым материалом типа 1, обуславливая анизотропию ударной вязкости. Практически одинаковое распределение полюсной плотности базиса в направлении прокатки и поперечном направлении определяет изотропность ударной вязкости в слоистом материале типа 2. Сравнительная оценка результатов испытаний показала, что при распространении трещины одновременно через все слои (Р-образец) более высокой ударной вязкостью характеризуется 7-ми слойный материал, а при распространении трещины последовательно через каждый слой 
(Т-образец) - 13-ти слойный материал. Количественная оценка характеристик разрушения позволила установить, что снижение ударной вязкости в Р-образцах с увеличением количества слоев обусловлено уменьшением как работы зарождения, так и работы распространения трещины. При этом повышение сопротивления ударного разрушения в Т-образцах происходит за счет существенного увеличения работы зарождения трещины при незначительном уменьшении ее работы распространения.

Ключевые слова: слоистый материал, титановый сплав, диффузионная сварка, текстура, ударная вязкость.

\section{1. Введение}

Слоистые металлические материалы (СМ), состоящие как из однородных, так и из разнородных материалов, привлекают к себе огромное внимание благодаря возможности достижения уникального комплекса свойств. Среди перспективных материалов, составляющих СМ, являются титан и его сплавы. Двухфазный титановый сплав ВТ6 широко применяется в аэрокосмической, судостроительной, нефтехимической и автомобильной промышленностях благодаря хорошей коррозионной стойкости, высокой удельной прочности, проявлению эффекта сверхпластичности и превосходной диффузионной свариваемости [1-7].

Диффузионная сварка (ДС) представляет собой процесс твердофазного соединения, при котором между двумя контактирующими поверхностями образуются связи на атомном уровне вследствие микроскопической пластической деформации и диффузии атомов. Многочисленные исследования направлены на оптимизацию процесса ДС с целью получения соединения без дефектов (поры/несплошности, остатки оксидной пленки), поскольку установлено отрицательное влияние последних на механические свойства соединений [4-7]. С другой стороны, ослабленные за счет пор соединения могут быть эффективными барьерами на пути распространения трещины в слоистом материале, полученном ДС. Так в работе [8] установлено, что в слоистом титановом материале вблизи границы раздела с несваренными зонами происходит замедление роста усталостной трещины. В работе [9] показано, что поры в зоне соединения способствуют многократному повышению ударной вязкости 13-ти слойного материала на основе сплава ВТ6 при распространении трещины последовательно через каждый слой. Стоит отметить, что данное исследование авторы проводили на СМ с изотропными свойствами, полученном благодаря определенной укладке листовых заготовок друг относительно друга [10].

Известно, что промышленные титановые сплавы характеризуются анизотропией свойств, которая в основном обусловлена кристаллографической текстурой [11-13]. Поскольку текстура материала и конструкций оказывает существенное влияние на их технологические и эксплуатационные свойства $[13,14,15,16]$, представляется актуальным проведение текстурных исследований CM, полученного диффузионной сваркой титанового сплава ВТ6. Не менее актуальным и интересным направлением в отношении изучения данного материала является установления влияния количества слоев на его механическое поведение. В литературе данный вопрос изучен в основном на композитах, а также СМ на основе сталей, полученных методом горячей пакетной прокатки. В свою очередь отсутствие этих данных сдерживает разработку как однородных, так и разнородных СМ на основе титановых сплавов, которые могут быть применены также при разработке броневых элементов [17].

Настоящая работа является продолжением исследований механического поведения слоистого титанового материала, полученного ДС, и направлена на установление способов управления механическим поведением при ударном нагружении на стадии их получения.

\section{2. Материал и методика эксперимента}

В качестве исходного материала был выбран двухфазный титановый сплава ВТ6 производства ОАО «Корпорация ВСМПО - АВИСМА» г. В. Салда, аналог зарубежного сплава Ti-6Al-4V. Для исследований путем диффузионной сварки были изготовлены слоистые материалы, состоящие из семи и тринадцати листовых заготовок толщиной $\sim 1.5$ и $~ 0.8$ мм, соответственно.

В СМ типа 1 заготовки при сборке в пакет укладывали друг относительно друга так, что направление прокатки (НП) в них совпадало, а в СМ типа 2 - не совпадало, соответственно угол между НП в соседних заготовках составлял $0^{\circ}$ и $90^{\circ}$. Процесс твердофазного соединения выполняли в электрической вакуумной печи ОКБ-8086 при температуре $900^{\circ} \mathrm{C}$, охлаждение вместе с печью. Подробная методика описана в работе [9].

Испытания на ударный изгиб проводили на стандартных образцах с размерами $10 \times 10 \times 55 \mathrm{mм}^{3}$ в соответствии с ГОСТ 9454. Образцы отличались расположением $\mathrm{U}$-образного надреза относительно поверхностей соединения (Рис. 1). В образце с “разветвляющим” трещину расположением поверхностей соединения (Р-образец) трещина распространяется одновременно через все слои (Рис. 1a), в образце с “тормозящим” трещину расположением поверхностей соединения (Т-образец) трещина распространяется последовательно через каждый слой (Рис. 1b) [18]. Испытания на ударный

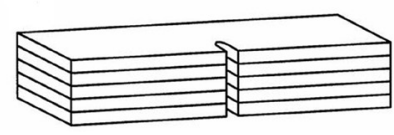

a

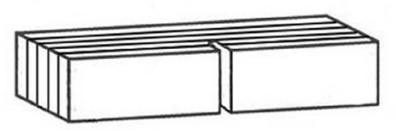

b
Рис. 1. Образец для испытаний на ударный изгиб с: "разветвляющим» (Р-образец) (а) и «тормозящим» (Т-образец) (b) трещину расположением поверхностей соединения.

Fig. 1. Sample for impact test: crack divider (a) and crack arrester (b) orientations. 
изгиб проводили при комнатной температуре на копре с падающим грузом Instron CEAST 9350 и записью диаграмм ударного нагружения в координатах «Усилие - перемещение». Разделение полной работы разрушения ударного образца на ее составляющие - работу зарождения трещины $\left(A_{3}\right)$ и работу распространения трещины $\left(A_{\mathrm{p}}\right)$ осуществляли согласно [19]. Погрешность измерений величин ударной вязкости не превышала $2 \%$.

Металлографические и фрактографические исследования проводили на растровом электронном микроскопе «TESCAN MIRA3 LMU».

Для изучения текстуры использовали метод обратных полюсных фигур (ОПФ), построенных для а-фазы сплава ВТ6 по методу Вильсона [20]. ОПФ строили для плоскостей листа, которые позволяли оценивать плотность в НП, в поперечном направлении (ПН) и в нормальном направлении (НH) к плоскости прокатки.

\section{3. Результаты и обсуждение}

На Рис. 2 и в Табл. 1 представлены результаты металлографических исследований сплава ВТ6 в виде листа толщиной $\sim 0.8$ мм в состоянии поставки и после ДС. В плоскости прокатки зерна а-фазы имеют равноосную форму, их средний размер составляет 3.6 мкм. В продольном сечении зерна $\alpha$-фазы вытянуты в направлении деформации. В результате ДС при температуре $900^{\circ} \mathrm{C}$ происходит небольшой рост зерен и снижение степени вытянутости а-фазы с 1.6 до $\sim 1.1$. Согласно ранее опубликованным данным [9] в зоне соединения присутствуют единичные поры сферической формы размером менее 1 мкм.

Результаты испытаний показали (Табл. 2), что СM, в котором угол между НП в соседних заготовках составляет $0^{\circ}$, характеризуются анизотропией ударной

Табл. 1. Размер зерен $\alpha$-фазы сплава ВТ6 (лист толщиной 0.8 мм) до и после ДС.

Table 1. Grain sizes of the $\alpha$-phase of the Ti-6Al- $4 \mathrm{~V}$ alloy (sheet of $\sim 0.8 \mathrm{~mm}$ thickness) before and after DB.

\begin{tabular}{|c|c|c|c|}
\hline \multirow{2}{*}{$\begin{array}{l}\text { Плоскости } \\
\text { Planes }\end{array}$} & \multirow{2}{*}{$\begin{array}{c}\text { Направление измерения } \\
\text { относительно вытянутости зерен } \\
\text { Direction of measurement } \\
\text { relative to grain elongation }\end{array}$} & \multicolumn{2}{|c|}{$\begin{array}{l}\text { Размер зерен } \alpha \text {-фазы, мкм } \\
\text { Grain size of the } \alpha \text { phase, } \mu \mathrm{m}\end{array}$} \\
\hline & & $\begin{array}{c}\text { Состояние поставки } \\
\text { As-received state }\end{array}$ & $\begin{array}{c}\text { Состояние после ДС } \\
\text { State after DB }\end{array}$ \\
\hline \multirow{2}{*}{$\begin{array}{l}\text { Продольное сечение } \\
\text { Longitudinal section }\end{array}$} & Вдоль / Along & $4.0 \pm 0.5$ & $5.2 \pm 0.4$ \\
\hline & Поперек / Across & $2.5 \pm 0.4$ & $4.7 \pm 0.3$ \\
\hline \multirow{2}{*}{$\begin{array}{l}\text { Поперечное сечение } \\
\text { Cross section }\end{array}$} & Вдоль / Along & $3.9 \pm 0.5$ & $5.4 \pm 0.3$ \\
\hline & Поперек /Across & $2.7 \pm 0.3$ & $4.9 \pm 0.4$ \\
\hline \multirow{2}{*}{$\begin{array}{l}\text { Плоскость прокатки } \\
\text { Rolling plane }\end{array}$} & Вдоль / Along & $3.6 \pm 0.5$ & $4.8 \pm 0.6$ \\
\hline & Поперек / Across & $3.6 \pm 0.5$ & $4.8 \pm 0.6$ \\
\hline
\end{tabular}

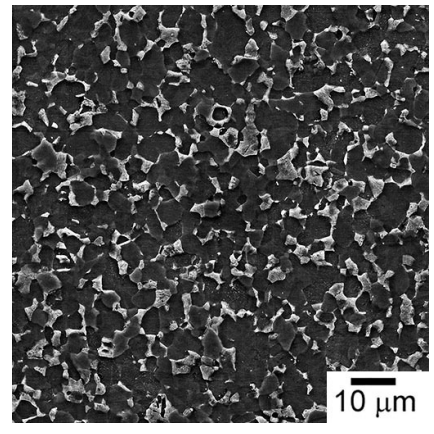

a

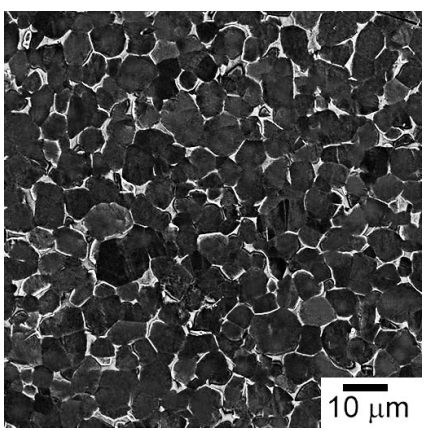

d

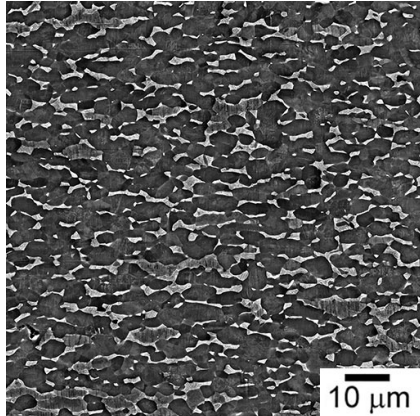

$\mathrm{b}$

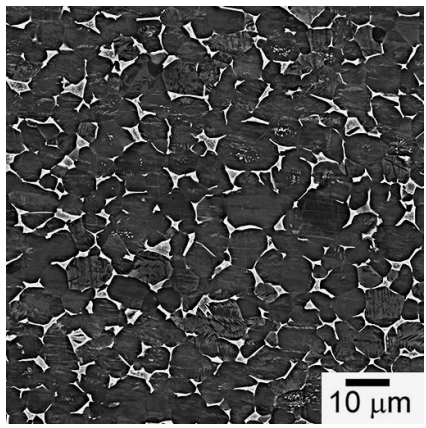

e
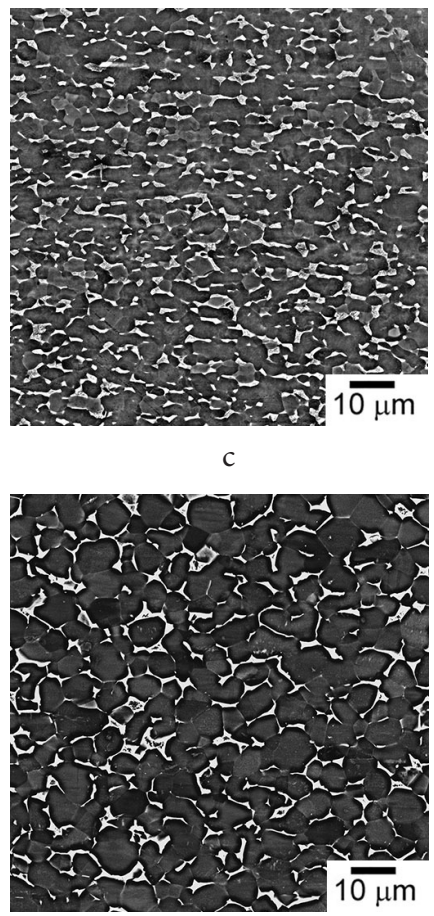

Pис. 2. Микроструктура сплава ВТ6 (лист толщиной 0.8 мм) до (a-c) и после ДС (d-f): плоскость прокатки (a,d), продольное сечение (b,e), поперечное сечение (c,f).

Fig. 2. Microstructure of the Ti-6Al-4V alloy (sheet of $\sim 0.8 \mathrm{~mm}$ thickness) before (a-c) and after DB (d-f): rolling plane (a,d), longitudinal section $(\mathrm{b}, \mathrm{e})$, cross section $(\mathrm{c}, \mathrm{f})$. 
вязкости в двух взаимно-перпендикулярных направлениях. Материал обладает повышенным сопротивлением разрушению при распространении трещины одновременно через все слои. При этом Р-образец, вырезанный поперек направления прокатки, обладает максимальным значением ударной вязкости, равным 0.73 МДж/м².

Как показано в работе [10] укладка листовых заготовок друг относительно друга так, что угол между НП в соседних заготовках составляет $90^{\circ}$, способствуют получению СМ с изотропными свойствами. Ударная вязкость 13-ти слойного материала составляла 0.66 МДж/м ${ }^{2}$

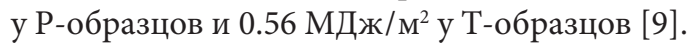

Результаты текстурных исследований показали, что листы в состоянии поставки имеют текстуру призматического типа (11 $\overline{2} 0)[10 \overline{1} 0]$. Данная текстура наследуется слоистым материалом типа 1 (НП в заготовках совпадает), обуславливая анизотропию ударной вязкости (Табл. 2). Повышенную полюсную плотность в плоскости прокатки имеет плоскость призмы $(11 \overline{2} 0)$,

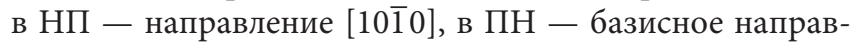
ление [0001] (Рис. 3а). При этом полюсная плотность соответственно равна 5.2, 3.7 и 8.01. В слоистом материале типа 2, состоящем из листовых заготовок, расположенных друг относительно друга так, что угол между НП в соседних заготовках составляет $90^{\circ}$, повышенную полюсную плотность в плоскости прокатки имеет плоскость призмы $(11 \overline{2} 0)$, в НП и ПН - направление [0001] (Рис. 3b). Полюсная плотность базиса в НП составляет 5.41, в ПН - 4.84. Практически одинаковое распределение полюсной плотности базиса в НП и ПН определяет изотропность ударной вязкости в данном слоистом материале (Табл. 2).

В [15,21] авторы показали успешное применение технологического метода устранения анизотропии и принципа слоистости при изготовлении сферического сосуда давления.

Табл. 2. Ударная вязкость образцов 13-ти слойного материала типа 1 с «разветвляющим» (Р) и «тормозящим» (Т) трещину расположением поверхностей соединения.

Table 2. The impact strength of samples of the 13-layer material of type 1 in the crack divider and arrester orientations.

\begin{tabular}{|c|c|c|}
\hline \multirow{3}{*}{ Образец / Sample } & \multicolumn{2}{|c|}{ 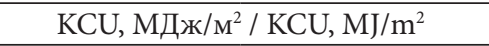 } \\
\hline & \multicolumn{2}{|c|}{$\begin{array}{c}\text { Направление вырезки образцов } \\
\text { Cutting direction of samples }\end{array}$} \\
\hline & Вдоль НП / RD & Поперек НП / TD \\
\hline $\mathrm{P} /$ crack divider & 0.57 & 0.73 \\
\hline $\mathrm{T} /$ crack arrester & 0.6 & 0.5 \\
\hline
\end{tabular}

Еще одним простым способом управления свойствами СМ является использование разного количества составляющих заготовок. Для этих целей был изготовлен 7-ми слойный материал типа 2 и проведен сравнительный анализ экспериментальных данных с ранее полученными данными для 13-ти слойного материала, полученного при аналогичных условиях [9].

Сравнительный анализ полученных данных (Табл. 3) показал, что при распространении трещины одновременно через все слои более высокой ударной вязкостью характеризуется 7-ми слойный образец, а при распространении трещины последовательно через каждый слой - 13-ти слойный образец. Полученные результаты согласуются с литературными данными как для клееных CM [22], так и полученных горячей пакетной прокаткой [23]. Механическое поведение 7-ми слойного материала качественно подобно поведению 13-ти слойного материала (Табл. 3). Образец с «разветвляющим» трещину расположением поверхностей соединения по сравнению с образцом с «тормозящим» трещину расположением поверхностей соединения характеризуется
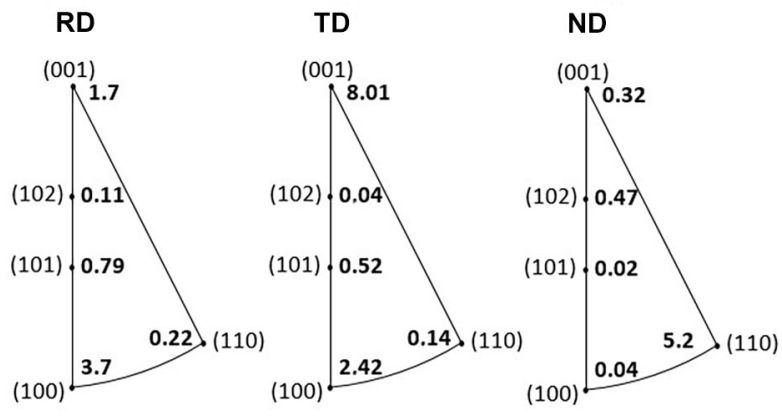

a
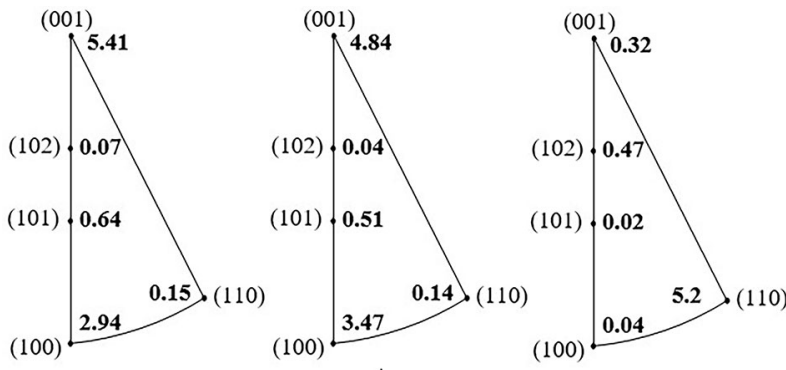

$\mathrm{b}$

Рис. 3. Обратные полюсные фигуры в слоистом материале типа 1 (a) и типа 2 (b).

Fig. 3. Reverse pole figures in the layered material type 1 (a) and type 2 (b).

Табл. 3. Ударная вязкость (KCU), работа зарождения трещины $\left(A_{3}\right)$ и работа распространения трещины $\left(A_{\mathrm{p}}\right)$ в образцах с «разветвляющим» $(\mathrm{P})$ и «тормозящим» $(\mathrm{T})$ трещину расположением поверхностей соединения.

Table 3. The impact strength (KCU), the crack initiation energy $\left(A_{\mathrm{i}}\right)$ and the crack propagation energy $\left(A_{\mathrm{pr}}\right)$ of samples in the crack divider and arrester orientations.

\begin{tabular}{|c|c|c|c|c|c|c|}
\hline \multirow[b]{2}{*}{$\begin{array}{l}\text { Количество слоев } \\
\text { Number of layers }\end{array}$} & \multicolumn{2}{|c|}{ 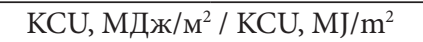 } & \multicolumn{2}{|c|}{$A_{3}$, Дж / $A_{\mathrm{i}}, \mathrm{J}$} & \multicolumn{2}{|c|}{$A_{\mathrm{p}}$, Дж / $A_{\mathrm{pr}}, \mathrm{J}$} \\
\hline & $\begin{array}{c}\text { Р-образец } \\
\text { crack divider }\end{array}$ & $\begin{array}{c}\text { T-образец } \\
\text { crack arrester }\end{array}$ & $\begin{array}{c}\text { Р-образец } \\
\text { crack divider }\end{array}$ & $\begin{array}{c}\text { Т-образец } \\
\text { crack arrester }\end{array}$ & $\begin{array}{c}\text { Р-образец } \\
\text { crack divider }\end{array}$ & $\begin{array}{c}\text { Т-образец } \\
\text { crack arrester }\end{array}$ \\
\hline 7 & 0.81 & 0.47 & 23.1 & 22.4 & 39.6 & 13.8 \\
\hline $13[9]$ & 0.66 & 0.56 & 19.5 & 19.6 & 34.8 & 21.4 \\
\hline
\end{tabular}




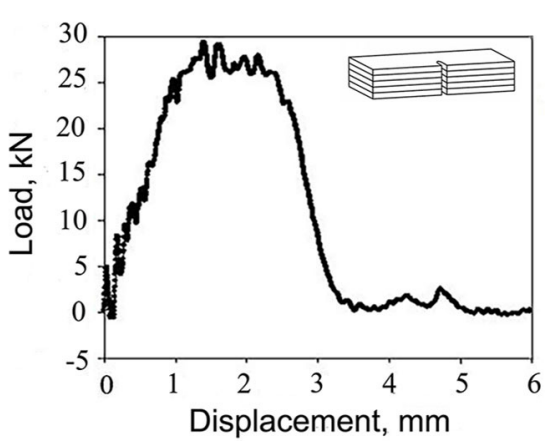

a

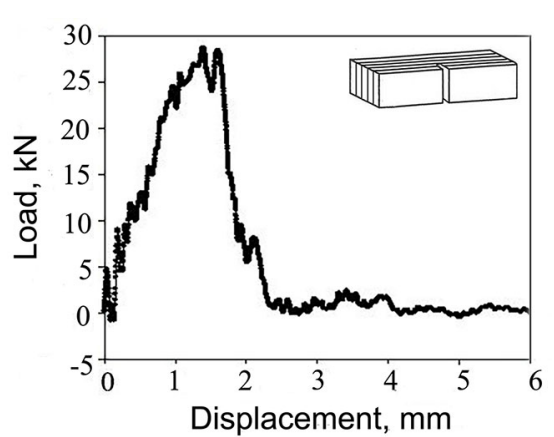

$\mathrm{b}$

Рис. 4. Диаграммы ударного нагружения 7-ми слойного материала типа 2. Р-образец (a), Т-образец (b).

Fig. 4. Impact loading diagrams of 7-layer material type 2. Crack divider (a) and crack arrester (b) orientations.

повышенной ударной вязкостью, равной 0.81 МДж/м². При этом работа зарождения трещины одинаковая, a еe работа распространения выше для Р-образца, что и обуславливает его повышенное сопротивлению разрушению. Известно [24], что энергоемкость разрушения определяется радиусом пластической зоны, окаймляющей край трещины. Поэтому, возможно, что более высокое значение ударной вязкости Р-образца по сравнению с Т-образцом связано со значительным размером пластической зоны в вершине U-образного надреза, захватывающей сразу несколько слоев испытываемого образца.

По диаграммам ударного нагружения видно (Рис. 4), что вид кривых до достижения максимума нагрузки является одинаковым для всех исследованных образцов, а после - наблюдается более ускоренный спад нагрузки в Т-образце, что свидетельствует о быстрой скорости распространения трещины.

С увеличением количества слоев наблюдаемое снижение ударной вязкости в Р-образцах обусловлено как за счет снижения работы зарождения, так и распространения трещины (Табл. 3). Повышение ударной вязкости в Т-образцах происходит благодаря значительному увеличению работы распространения трещины при незначительном уменьшении работы зарождения трещины.

\section{4. Заключение}

В данной работе показаны способы управления механическим поведением слоистого материала из титанового сплава ВТ6, полученного диффузионной сваркой. По результатам проведенных исследований сделаны следующие выводы:

1. Анизотропия ударной вязкости слоистого материала, состоящего из листовых заготовок, расположенных друг относительно друга так, что направление прокатки в них совпадает, определяется призматической текстурой. Изотропность свойств слоистого материала, состоящего из заготовок, расположенных друг относительно друга так, что направление прокатки в соседних заготовках не совпадает, обусловлена практически одинаковым распределением полюсной плотности базиса в направлении прокатки и поперечным ему направлении.
2. Семислойный материал характеризуется наибольшим сопротивлением ударному разрушению при «разветвляющем» трещину расположении поверхностей соединения (Р-образец), что обусловлено повышенной работой распространения трещины.

3. С увеличением количества слоев ударная вязкость материала при «тормозящем» трещину расположении поверхностей соединения (Т-образец) повышается, а при «разветвляющем» трещину расположении поверхностей соединения (Р-образец) - понижается.

Благодарности/Acknowledgements. Aвтор выражает благодарность Круглову А. А. за помощь при изготовлении слоистых материалов, а также Даниленко В.Н. за помощьв в проведении текстурных исследований. Работа выполнена в рамках государственного задания ИПСМ РАН № АAАA-A17117041310221 5. Микроструктурные и рентгеноструктурные исследования проводились на базе ЦКП ИПСМ РАН, механические испьтания на ударный изгиб - на базе ЦКП «НАНОТЕХ» УГАТУ. / The author is grateful to A.A. Kruglov for help in the manufacture of layers materials, as well as Danilenko V.N. for help with texture research. The present work was accomplished according to the state assignment of IMSP RAS №. AAAA-A17117041310221 5. Microstructural and X-ray studies were carried out in the CCU of IMSP RAS, mechanical impact test - in the CCU of "NANOTECH" USATU.

\section{Литература/References}

1. Titanium and Titanium Alloys: Fundamentals and Applications. 1rd edn (ed. by C. Leyens, M. Peters). Weinheim, Wiley-VCH (2003) 523 p. Crossref

2. R.V. Safiullin, R.M. Galeyev, O.R. Valiakhmetov et al. Lett. Mater. 6 (4), 281 (2016). (in Russian) [А. Р. Сафиуллин, Р. М. Галеев, О. Р. Валиахметов и др. Письма о материалах. 6 (4), 281 (2016).] Crossref

3. R. V. Safiullin, M.H. Muhametrahimov, S.P. Malycheva et al. Lett. Mater. 8 (3), 329 (2018). (in Russian) [Р. В. Сафиуллин, М. Х. Мухаметрахимов, С.П. Малышева и др. Письма о материалах. 8 (3), 329 (2018).] Crossref

4. H.-S. Lee. In: Welding and Joining of Aerospace Materials (ed.by M.C. Chaturvedi). Sawston, Woodhead Publishing Limited (2012) p. 320. 
5. E. Yakushina, A. Reshetov, I. Semenova et al. Materials Science and Engineering: A. 726, 251 (2018). Crossref

6. F. A. Calvo, J. M. Gómez De Salazar et al. J. Mater. Sci. 27, 391 (1992). Crossref

7. R. Ya. Lutfullin. Lett. Mater. 1 (1), 59 (2011). (in Russian) [Р.Я. Лутфуллин. Письма о материалах. 1 (1), 59 (2011).] Crossref

8. X. He, Y. Dong, Y. Li, X. Wang. Int. J. Fatig. 106, 1 (2018). Crossref

9. A.A. Sarkeeva, A.A. Kruglov, R. Ya. Lutfullin et al. Composites Part B. 187, 107838 (2020). Crossref

10. A. A. Ganeeva, A. A. Kruglov, R. Y. Lutfullin. Rev. Adv. Mater. Sci. 25, 136 (2010).

11. P. G. Miklyaev, Ya.B. Fridman. Anisotropy of mechanical properties of metals. Moscow, Metallurgy (1986) 224 p. (in Russian) [П. Г. Микляев, Я.Б. Фридман. Анизотропия механических свойств металлов. 2-е изд. Москва, Металлургия (1986) 224 с.]

12. M. R. Bache, W. J. Evans. Mater. Sci. Eng. A. 319-321, 409 (2001). Crossref

13. S. Ya. Betsofen, A. A. Ilyin, S. V. Skvortsova et al. Metally. 2, 54 (2005). (in Russian) [С. Я. Бецофен, А. А. Ильин, С. В. Скворцова и др. Металлы. 2, 54 (2005).]

14. S. Ya. Betsofen, A. A. Ilyin, V. V. Plikhunov et al. Metally. 5, 51 (2007). (in Russian) [С. Я. Бецофен, А. А. Ильин, В. В. Плихунов и др. Металлы. 5, 51 (2007).

15. Patent RF № 2380185, 27.01.2010. (in Russian) [Патент РФ № 2380185, 27.01.2010.]

16. A.I. Horev. Svarochnoe proizvodstvo. 10, 11 (2012). (in Russian) [А. И. Хорев. Сварочное производство. 10, 11 (2012).]

17. A. I. Horev. Tekhnologiya legkih splavov. 4, 92 (2002). (in
Russian) [А.И. Хорев. Технология легких сплавов. 4, $92(2002)$.

18. J.D. Embury, N. J. Petch, A.E. Wraith et al. AIME MET SOC TRANS. 239 (1), 114 (1967).

19. M. Georgiev. Impact Crack-Resisting of Metals. Sofia, Bulvest 2000 (2007) 231 p. (in Bulgarian) [М. Георгиев. Пукнатиноустойчивост на металите при ударно натоварване. Софии, БУЛВЕСТ. 2000 (2007) 231 с.]

20. M. M. Borodkina, E. N. Spektor. X-ray analysis of the texture of metals and alloys. Moscow, Metallurgy (1981) 272 p. (in Russian) [М. М. Бородкина, Э.Н. Спектор. Рентгенографический анализ текстуры металлов и сплавов. Москва, Металлургия (1981) 272 с.]

21. A. A. Ganeeva, A. A. Kruglov, R. Y. Lutfullin. Deformaciya i razrushenie materialov. 7, 38 (2011). (in Russian) [А. А. Ганеева, А. А. Круглов, Р. Я. Лутфуллин. ДиРМ. 7, 38 (2011).]

22. G. I. Pogodin-Alekseev. Dynamic strength and brittleness of metals. Moscow, Mechanical Engineering (1966) 242 p. (in Russian) [Г.И. Погодин-Алексеев. Динамическая прочность и хрупкость металлов. Москва, Машиностроение (1966) 242 с.]

23. D. V. Vlasova, A.I. Plokhikh, A.A. Minakov. Novye materialy i tekhnologii v mashinostroenii. 27, 9 (2018). (in Russian) [Д. В. Власова, А.И. Плохих, А.А. Минаков. Новые материалы и технологии в машиностроении. 27, 9 (2018).]

24. K. Hellan. Introduction to fracture mechanics (translate from English A.S. Kravchuk; ed. by E. M. Morozov). Moscow, Mir (1988) 364 p. (in Russian) [К. Хеллан. Введение в механику разрушения (пер. с англ. А.С. Кравчука; под ред. Е.М. Морозова). Москва, Мир (1988) 364 с.] 